\title{
OPTIMAL CONTROL OF A PARTIALLY KNOWN COUPLED SYSTEM OF BOD AND DO
}

\section{LAOUAR CHAFIA ${ }^{1,2, *}$, AYADI ABDELHAMID ${ }^{1}$ AND HAFDALLAH ABDELHAK ${ }^{3}$}

${ }^{1}$ Laboratoire des Systèmes Dynamiques et Contrôle, Larbi Ben M'hidi University, Oum El Bouaghi, Algeria ${ }^{2}$ LAbbes Laghrour University, BP 1252 Route of Batna Khenchela, 40004 khenchela, Algeria ${ }^{3}$ Laboratory of Mathematics, Informatics and Systems (LAMIS), Larbi Tebessi University, Tébessa, Algeria

${ }^{*}$ Corresponding author: laouar_chafia@univ-khenchela.dz

\begin{abstract}
The work presented in this paper is concerned with the organic pollution problem and water quality valuation. Biochemical oxygen demand has been used to evaluate the quality of water. If organic matter is present the dissolved oxygen is consumed. This article considers an optimal control problem of coupled system with missing initial conditions, which presents the relation between the biochemical oxygen demand and the dissolved oxygen. The main objective is to control the concentration of dissolved oxygen using the information given in the biochemical oxygen demand equation. The main tool used to characterize the optimal control of the investigate system under the Pareto control formulation.
\end{abstract}

\section{INTRODUCTION}

The environmental pollution problem is one most serious problems faced by the world. It is always linked to some terrible problems, which are unable to find a solution and causing irreparable nature damage. The presence of a sufficient concentration of dissolved oxygen (DO) is all-important and necessary to preserve water life. If more oxygen is consumed than is produced, dissolved oxygen levels decline and some sensitive animals may move away, weaken, or die. Oxygen is gained from the atmosphere and plants as a result of photosynthesis. Running water, because of its churning, dissolves more oxygen than still water. Respiration

Received August 23 ${ }^{\text {rd }}, 2021$; accepted October $7^{\text {th }}, 2021$; published November 25 $5^{\text {th }}, 2021$.

2010 Mathematics Subject Classification. 49J21, 93C20, 93C41.

Key words and phrases. optimal control; problem with missing data; pareto control; no regret control; least regret controls; optimality system.

(C)2021 Authors retain the copyrights of their papers, and all open access articles are distributed under the terms of the Creative Commons Attribution License. 
by aquatic animals, decomposition, and various chemical reactions consume oxygen. The required quantity of dissolved oxygen by aerobic biological organisms which is used for decomposing organic material under aerobic conditions at a specified temperature is called Biochemical oxygen demand (BOD). The decrease of BOD is the way for judging the effectiveness of water purification [1-3]. Many studies have been published in the context of improving the quality of the method and procedure can use to reduce the BOD level, we refer to works by D.M. Reynolds, S.R. Ahmad in [12], Salguero, Jazmin and Valverde, Jhonny in [13], Magdalena Zajda and Urszula Aleksander-Kwaterczak in [14], etc.

This article provides the main insights into the debate on optimal control choice of an evolution coupled system that presents the relation between biochemical oxygen demand and dissolved oxygen. Because the concentration of dissolved oxygen is of prime importance in considering the quality of water, we try to control its level by giving an assessment of the biochemical oxygen demand and for studying too its physicochemical characteristics. Elsewhere, the posed coupled systems are given with unknown initial conditions that present some barriers. The main aim of our work is to characterize the optimal control. For finding the characterization of this optimal control, we dispose of the incomplete data by introducing the concepts of no-regret control and the sequence of least regret controls. The optimality coupled systems of the no regret control are formed by passing to the limit.

\section{Setting the Problem}

In this section, we present a mathematical model that is used for studying the pollution problem. This considered model is not standard because it contains some missing initial conditions.

We consider a fixed final time $T>0$, and $\Omega$ a bounded open subset of $\mathbb{R}^{N}, N \in 1,2,3$ of smooth boundary $\Gamma$. We denote by $Q=\Omega \times] 0, T[$ the space-time cylinder and by $\Sigma=\Gamma \times] 0, T[$ her boundary. We are interested in an evolutionary organic pollution problem in surface waters for example lakes or estuaries which is reduced to this reaction-dispersion/diffusion problem with uncertainly

$$
\left\{\begin{array}{cc}
\frac{\partial y}{\partial t}-\mathrm{d} i v(d(x) \nabla y)+r(x) y=0 & \text { in } \quad Q \\
\frac{\partial z}{\partial t}-\mathrm{d} i v(d(x) \nabla z)+\tilde{r}(x) z+r(x) y=\omega \chi_{\mathcal{O}} & \text { in } \quad \Omega \\
y(x, 0)=g_{1}, z(x, 0)=g_{2} & \text { on } \quad \Sigma \\
z=0, \frac{\partial z}{\partial \nu}=0 &
\end{array}\right.
$$

where $y, z$ are BOD and DO in a given water sample at a certain temperature over a specific time period. The control function $\omega$ presents the sources of dissolved oxygen from the atmosphere and photosynthesis of plants on the control region $\mathcal{O}$, and $\chi_{\mathcal{O}}$ is the characteristic function of $\mathcal{O}$. We suppose that for all $\omega \in \mathcal{U}_{a d}$, we have

$$
\mathcal{U}_{a d}=\left\{\omega \in L^{2}(Q): \omega_{\min } \leq \omega \leq \omega_{\max }\right\} \text { is non-empty closed, convex }
$$


were $\omega_{\min }$ and $\omega_{\max }$ present the minimum and the maximum concentrations of dissolved oxygen that would be present in water at a specific temperature, in the absence of other factors. The initial conditions $\left(g_{1}, g_{2}\right) \in$ $\mathcal{G} \subset H^{-\frac{1}{2}}(\Omega) \times H_{0}^{1}(\Omega)$ assumed to be unknown. The boundary conditions $\left(z, \frac{\partial z}{\partial \nu}\right) \in H^{-\frac{1}{2}}(\Sigma) \times H^{-\frac{1}{2}}(\Sigma)$. The functions $r, \tilde{r}$ and $d$ are reaction coefficients.

The coupled systems $(2.1)$ has a unique pair solution $(y, g)=(y(\omega, g), z(\omega, g))$ where

$$
(y, z) \in L^{2}(Q) \cap \mathcal{C}^{\infty}\left(0, T ; H^{-\frac{1}{2}}(\Omega)\right) \times L^{2}\left(0, T ; H_{0}^{1}(\Omega) \cap H^{2}(\Omega)\right) \cap \mathcal{C}^{\infty}\left(0, T ; H_{0}^{1}(\Omega)\right)
$$

There are many factors that can be reduced the level of dissolved oxygen like the respiration of the plant life and the animal life, decomposition of organic matter, reduction due to other gases, temperature increase, and others. For these reasons, the main goal of this work is to control the evolutionary organic pollution problem. Exactly, we control the level of dissolved oxygen, where we point out here that we did not insert any control function in the biochemical oxygen demand equations.

For fixed pair $\left(y_{d}, z_{d}\right) \in\left(L^{2}(\Omega)\right)^{2}$ and for $N>0, g=\left(g_{1}, g_{2}\right) \in \mathcal{G}$, we define the quadratic cost function associated to $(2.1)$

$$
J(\omega, g)=\left\|y(\omega, g)-y_{d}\right\|_{L^{2}(Q)}^{2}+\left\|z(\omega, g)-z_{d}\right\|_{L^{2}(Q)}^{2}+\int_{0}^{T} \int_{\mathcal{O}} N \omega^{2} \mathrm{~d} t \mathrm{~d} x .
$$

Let us minimize the following optimal control with incomplete data:

$$
\inf _{\omega \in \mathcal{U}_{a d}} J(\omega, g) \forall g \in \mathcal{G}
$$

The problem (2.4) has no sense in the case of the space $\mathcal{G}$ is infinite. Then, if $\mathcal{G}$ is finite we try to solve the inf - sup problem

$$
\inf _{\omega \in \mathcal{U}_{a d}} \sup _{g \in \mathcal{G}} J(\omega, g) .
$$

However, in this situation, it is very difficult to ensure that $\sup _{g \in \mathcal{G}} J(\omega, g)$ is bounded.

In (1992), J.L.Lions has done a good idea by adding an additional concept which is called "no regret control. The concept of no-regret control (or, equivalently, Pareto control) of distributed systems with missing data is used by J.L. Lions in [4, Pareto control of distributed systems, page 90]. In [4-7], J.L. Lions applied the Pareto control and he associated it with a sequence of low-regret controls defined by a quadratic perturbation for deterministic distributed systems with incomplete data. In [10], O. Nakolima, R. Dorville, and A. Omrane studied how the no regret control can be extended to the hyperbolic case. They also generalized these concepts in the case of ill-posed deterministic problems, without assuming Slater's condition [8,9]. In [11], Hafdallah A, and Ayadi A applied no regret and low regret concepts to control a thermoelastic body with missing initial conditions. A. Hafdallah, A. Ayadi, and C. Laouar applied the no-regret control notion to control an ill-posed wave equation, see [15]. 
The principle of this idea is based on looking for controls such that

$$
J(\omega, g) \leq J(0, g) \forall g \in \mathcal{G}
$$

Condition (2.6) implies

$$
\sup _{g \in \mathcal{G}}[J(\omega, g)-J(0, g)] \text { is bounded. }
$$

In that case, we solve the following problem

$$
\inf _{\omega \in \mathcal{U}_{a d}} \sup _{g \in \mathcal{G}}[J(\omega, g)-J(0, g)]
$$

In the following, we are defining the no regret control for the partially known problem (2.1).

\section{Defining the NO-REgRet CONTROL}

We say that $\hat{\omega} \in \mathcal{U}_{a d}$ defines a no-regret control for (2.1) if it is the optimal solution of (2.7).

Lemma 3.1. For every $\omega \in \mathcal{U}_{a d}$ the problem (2.7) is equivalent to

$$
\inf _{\omega \in \mathcal{U}_{a d}}\left(J(\omega, 0)-J(0,0)+2 \sup _{g \in \mathcal{G}} \int_{\Omega}\left[g_{1} \cdot \zeta(\omega)(x, 0)+g_{2} \cdot \xi(\omega)(x, 0)\right] \mathrm{d} x, g=\left(g_{1}, g_{2}\right) \in \mathcal{G},\right.
$$

where $(\zeta, \xi)=(\zeta(\omega, 0)(x, t), \xi(\omega, 0)(x, t))$ satisfies the following backward coupled equations

$$
\left\{\begin{array}{cc}
-\frac{\partial \zeta}{\partial t}-\mathrm{d} i v(d(x) \nabla \zeta)+r(x) \zeta+r(x) \xi=y(\omega, 0) & \text { in } \quad Q \\
-\frac{\partial \xi}{\partial t}-\mathrm{d} i v(d(x) \nabla \xi)+\tilde{r}(x) \xi=z(\omega, 0) & \\
\zeta(x, T)=0, \xi(x, T)=0 & \text { in } \quad \Omega \\
\zeta=0, \frac{\partial \zeta}{\partial \nu}=0 & \text { on } \quad \Sigma
\end{array}\right.
$$

Proof. By linearity, we can write the solution to $(2.7)$ in the form $y(\omega, g)=y(\omega, 0)+y(0, g), z(\omega, g)=$ $z(\omega, 0)+z(0, g)$. Then, the functional $J(\omega, g)$ can be written

$$
J(\omega, g)=J(\omega, 0)-J(0,0)+2 \iint_{Q}[y(\omega, 0) y(0, g)+z(\omega, 0) z(0, g)] \mathrm{d} t \mathrm{~d} x .
$$

We introduce $(\zeta(\omega), \xi(\omega))$ the solution of $(3.2)$. Then, we use integration by parts, we obtain

$$
\begin{aligned}
\iint_{Q}-\left(\frac{\partial \zeta}{\partial t}+\mathrm{d} i v(d(x) \nabla \zeta)+r(x) \zeta+r(x) \xi\right) y(0, g) \mathrm{d} t \mathrm{~d} x & \\
& =\int_{\Omega} g_{1} \zeta(\omega)(x, 0) \mathrm{d} x+\iint_{Q} \xi r(x) y(0, g) \mathrm{d} t \mathrm{~d} x
\end{aligned}
$$

and

$$
\begin{aligned}
\iint_{Q}\left(-\frac{\partial \xi}{\partial t}-\mathrm{d} i v(d(x) \nabla \xi)+\tilde{r}(x) \xi\right) z(0, g) \mathrm{d} t \mathrm{~d} x \\
=\int_{\Omega} g_{2} \cdot \xi(\omega)(x, 0) \mathrm{d} x+\iint_{Q} \xi\left(\frac{\partial z}{\partial t}(0, g)-\mathrm{d} i v(d(x) \nabla z(0, g))+\tilde{r}(x) z(0, g)\right) \mathrm{d} t \mathrm{~d} x
\end{aligned}
$$


Adding (3.3) to (3.4), we get

$$
\iint_{Q}[y(\omega, 0) y(0, g)+z(\omega, 0) z(0, g)] \mathrm{d} t \mathrm{~d} x=\int_{\Omega}\left[g_{1} \cdot \zeta(\omega)(x, 0)+g_{2} \cdot \xi(\omega)(x, 0)\right] \mathrm{d} x .
$$

Remark 3.1. The no regret control exist only if $g_{1}$ and $\zeta(\omega)(x, 0)$ (respectively $g_{2}$ and $\zeta(\omega)(x, 0)$ ) are perpendicular to each other in $H^{-\frac{1}{2}}(\Omega)$ (respectively in $H_{0}^{1}(\Omega)$ ). For this reason, we consider the following set of admissible controls

$$
\hat{\mathcal{U}}_{a d}=\left\{\omega \in \mathcal{U}_{a d}:\left\langle g_{1}, \zeta(\omega)(x, 0)\right\rangle_{H^{-\frac{1}{2}}(\Omega)}=0,\left\langle g_{2}, \xi(\omega)(x, 0)\right\rangle_{H_{0}^{1}(\Omega)}=0\right\}
$$

In [4-7], J.L. Lions applied the no control and he associated it with a sequence of low-regret controls defined by a quadratic perturbation for deterministic distributed systems with incomplete data. The sequence of low-regret controls is expected to converge to the no regret control.

\section{Defining the Sequence of LOW-Regret controls (Least Regret controls)}

For every $\gamma>0$, we relax the problem (3.1) by introducing a quadratic perturbation such that

$$
J(\omega, g)-J(0, g) \leq \gamma\|g\|_{\mathcal{G}}^{2}, \forall g \in \mathcal{G} .
$$

We say that $\hat{\omega}^{\gamma} \in \mathcal{U}_{a d}$ is the sequence of low-regret controls for (2.1) if $\hat{\omega}^{\gamma}$ is the solution to

$$
\inf _{\omega \in \mathcal{U}_{a d}} \sup _{g \in \mathcal{G}}\left[J(\omega, g)-J(0, g)-\gamma\left(\left\|g_{1}\right\|_{H^{-\frac{1}{2}}(\Omega)}^{2}+\left\|g_{2}\right\|_{H_{0}^{1}(\Omega)}^{2}\right)\right] .
$$

Lemma 4.1. Problem (4.1) can be written as

$$
\inf _{\omega \in \mathcal{U}_{a d}} \mathcal{J}^{\gamma}(\omega)
$$

where

$$
\mathcal{J}^{\gamma}(\omega)=J(\omega, 0)-J(0,0)+\frac{1}{\gamma}\|\zeta(\omega)(x, 0)\|_{H^{-\frac{1}{2}}(\Omega)}^{2}+\frac{1}{\gamma}\|\xi(\omega)(x, 0)\|_{H_{0}^{1}(\Omega)}^{2} .
$$

Proof. From (3.1) and (3.2), the problem (4.1) is written as

$$
\inf _{\omega \in \mathcal{U}_{a d}}\left(J(\omega, 0)-J(0,0)+\sup _{g \in \mathcal{G}} \int_{\Omega}\left[\left(2 g_{1} \zeta(\omega)(x, 0)-\gamma g_{1}^{2}\right)+\left(2 g_{2} \xi(\omega)(x, 0)-\gamma g_{2}^{2}\right)\right] \mathrm{d} x\right) .
$$

The functions $f: g_{1} \mapsto\left(2 g_{1} \zeta(\omega)(x, 0)-\gamma g_{1}^{2}\right)$ and $\tilde{f}: g_{2} \mapsto\left(2 g_{2} \xi(\omega)(x, 0)-\gamma g_{2}^{2}\right)$ are concave. Then, it's absolutely clear that

$$
\sup _{g_{1} \in H^{-\frac{1}{2}}(\Omega)} f\left(g_{1}\right)=\frac{1}{\gamma}\|\zeta(\omega)(x, 0)\|_{H^{-\frac{1}{2}}(\Omega)}^{2}, \sup _{g_{2} \in H_{0}^{1}(\Omega)} \tilde{f}\left(g_{2}\right)=\frac{1}{\gamma}\|\xi(\omega)(x, 0)\|_{H_{0}^{1}(\Omega)}^{2} .
$$

Lemma 4.2. The problem (4.2)-(4.3) has a unique solution $\hat{\omega}^{\gamma}$, which called sequence of least regret controls. Furthermore, when $\gamma \rightarrow 0$, the control $\hat{\omega}^{\gamma}$ converges weakly to the unique no regret control $\hat{\omega}$. 
Proof. Since the set of admissible controls $\mathcal{U}_{a d}$ is non-empty closed and bounded, we have

$$
\mathcal{J}^{\gamma}(\omega) \geq-J(0,0)=-\left\|y_{d}\right\|_{L^{2}(\Omega)}^{2}-\left\|z_{d}\right\|_{L^{2}(\Omega)}^{2}, \forall \omega \in \mathcal{U}_{a d}
$$

Then there exists

$$
d^{\gamma}:=\inf _{\omega \in \mathcal{U}_{a d}} \mathcal{J}^{\gamma}(\omega) \geq 0
$$

Let $\left(\omega_{n}^{\gamma}\right) \in \mathcal{U}_{a d}$ be a minimizing sequence such that $d^{\gamma}=\lim _{n \rightarrow \infty} \mathcal{J}^{\gamma}\left(\omega_{n}^{\gamma}\right)=\mathcal{J}^{\gamma}\left(\omega^{\gamma}\right)$. Then, we get

$$
d^{\gamma} \leq \mathcal{J}^{\gamma}\left(\omega_{n}^{\gamma}\right)<d^{\gamma}+\frac{1}{n}<d^{\gamma}+1
$$

So, we deduce the bounds

$$
\begin{aligned}
\left\|\omega_{n}^{\gamma}\right\|_{L^{2}(0, T ; \mathcal{O})} \leq C^{\gamma},\left\|y\left(\omega_{n}^{\gamma}, 0\right)\right\|_{L^{2}(Q)} & \leq C^{\gamma},\left\|z\left(\omega_{n}^{\gamma}, 0\right)\right\|_{L^{2}(Q)} \leq C^{\gamma} \\
& \frac{1}{\sqrt{\gamma}}\left\|\zeta\left(\omega_{n}^{\gamma}\right)(x, 0)\right\|_{H^{-\frac{1}{2}}(\Omega)} \leq C^{\gamma}, \frac{1}{\sqrt{\gamma}}\left\|\xi\left(\omega_{n}^{\gamma}\right)(x, 0)\right\|_{H_{0}^{1}(\Omega)} \leq C^{\gamma} .
\end{aligned}
$$

where $C^{\gamma}$ is a positive constant and $\left(y_{n}^{\gamma}, z_{n}^{\gamma}\right)=\left(y\left(\omega_{n}^{\gamma}, 0\right), z\left(\omega_{n}^{\gamma}, 0\right)\right)$ solves the coupled systems

$$
\left\{\begin{array}{cc}
\frac{\partial y_{n}^{\gamma}}{\partial t}-\mathrm{d} i v\left(d(x) \nabla y_{n}^{\gamma}\right)+r(x) y_{n}^{\gamma}=0 & \text { in } \quad Q \\
\frac{\partial z_{n}^{\gamma}}{\partial t}-\mathrm{d} i v\left(d(x) \nabla z_{n}^{\gamma}\right)+\tilde{r}(x) z_{n}^{\gamma}+r(x) y_{n}^{\gamma}=\omega_{n}^{\gamma} \chi_{\mathcal{O}} & \\
y_{n}^{\gamma}(x, 0)=0, z_{n}^{\gamma}(x, 0)=0, & \text { in } \quad \Omega \\
z_{n}^{\gamma}=0, \frac{\partial z_{n}^{\gamma}}{\partial \nu}=0 & \text { on } \quad \Sigma
\end{array}\right.
$$

Multiplying the first equality of $(4.5)$ by $y_{n}^{\gamma}$ and the second equality by $z_{n}^{\gamma}$. We integrate over $\Omega$, we find

$$
\frac{1}{2} \frac{\mathrm{d}}{\mathrm{d} t} \int_{\Omega}\left|y_{n}^{\gamma}(t)\right|^{2} \mathrm{~d} x+\int_{\Omega} r(x)\left|y_{n}^{\gamma}(t)\right|^{2} \mathrm{~d} x-\int_{\Omega} \mathrm{d} i v\left(d(x) y_{n}^{\gamma}(t)\right) y_{n}^{\gamma}(t) \mathrm{d} x=0,
$$

and

$$
\frac{1}{2} \frac{\mathrm{d}}{\mathrm{d} t} \int_{\Omega}\left|z_{n}^{\gamma}(t)\right|^{2} \mathrm{~d} x+\int_{\Omega} \tilde{r}(x)\left|z_{n}^{\gamma}(t)\right|^{2}-\mathrm{d} i v\left(d(x) \cdot z_{n}^{\gamma}(t)\right) z_{n}^{\gamma}(t)+r(x) y_{n}^{\gamma}(t) z_{n}^{\gamma}(t) \mathrm{d} x
$$$$
=\int_{\mathcal{O}} \omega_{n}^{\gamma}(t) z_{n}^{\gamma}(t) \mathrm{d} x
$$

By integrating over $[0, T]$ and by applying the Gronwall lemma we obtain

$$
\left\|y_{n}^{\gamma}\right\|_{L^{\infty}(0, T ; \mathcal{Y})} \leq C^{\gamma},\left\|z_{n}^{\gamma}\right\|_{L^{\infty}\left(0, T ; H_{0}^{1}(\Omega)\right)} \leq C^{\gamma}
$$

where $C^{\gamma}$ is a positive constant. From (4.4), we deduce

$$
\left\|\frac{\partial z_{n}^{\gamma}}{\partial t}-\mathrm{d} i v\left(d(x) \nabla z_{n}^{\gamma}\right)+\tilde{r}(x) z_{n}^{\gamma}+r(x) y_{n}^{\gamma}\right\|_{L^{2}(0, T ; \mathcal{O})} \leq C^{\gamma}
$$


Then, there exists a subsequence of $\left(\omega_{n}^{\gamma}\right)$, that we denote with the same indices such that, when $n$ goes to $+\infty$,

$$
\begin{gathered}
\omega_{n}^{\gamma} \rightarrow \hat{\omega}^{\gamma} \text { weakly in } L^{2}(0, T ; \mathcal{O}), y_{n}^{\gamma} \rightarrow \hat{y}^{\gamma} \text { weakly in } L^{\infty}(0, T ; \mathcal{Y}), \\
z_{n}^{\gamma} \rightarrow \hat{z}^{\gamma} \text { weakly in } L^{\infty}\left(0, T ; H_{0}^{1}(\Omega)\right), \\
\frac{\partial z_{n}^{\gamma}}{\partial t}-\operatorname{div}\left(d(x) \nabla z_{n}^{\gamma}\right)+\tilde{r}(x) z_{n}^{\gamma}+r(x) y_{n}^{\gamma} \rightarrow f \text { weakly in } L^{2}(0, T ; \mathcal{O}) .
\end{gathered}
$$

The space $L^{\infty}(Q)$ (respectively $\left.L^{\infty}\left(0, T ; H_{0}^{1}(\Omega)\right)\right)$ is continuously embedded in $L^{2}(Q)$ (respectively $L^{2}\left(0, T ; H_{0}^{1}(\Omega)\right)$ ). Clearly, we have

$$
y_{n}^{\gamma} \rightarrow \hat{y}^{\gamma} \text { weakly in } L^{2}(Q), z_{n}^{\gamma} \rightarrow \hat{z}^{\gamma} \text { weakly in } L^{2}\left(0, T ; H_{0}^{1}(\Omega)\right)
$$

Multiplying two equalities in (4.5) by two test functions $\varphi, \psi \in D(Q)$, we obtain

$$
\begin{gathered}
\left\langle y_{n}^{\gamma},-\frac{\partial \varphi}{\partial t}-\operatorname{div}(d(x) \nabla \varphi)+r(x) \varphi\right\rangle_{L^{2}(Q)}=0 \\
\left\langle z_{n}^{\gamma},-\frac{\partial \psi}{\partial t}-\operatorname{div}(d(x) \nabla \psi)+\tilde{r}(x) \psi\right\rangle_{L^{2}(Q)}+\left\langle y_{n}^{\gamma}, r(x) \psi\right\rangle_{L^{2}(Q)}=\left\langle\omega_{n}^{\gamma}, \psi\right\rangle_{L^{2}(Q)} .
\end{gathered}
$$

By adding the last two equalities and passing to the limit, we get

$$
\left\{\begin{array}{c}
\frac{\partial \hat{y}^{\gamma}}{\partial t}-\operatorname{div}\left(d(x) \nabla \hat{y}^{\gamma}\right)+r(x) \hat{y}^{\gamma}=0 \\
\frac{\partial \hat{z}^{\gamma}}{\partial t}-\operatorname{div}\left(d(x) \nabla \hat{z}^{\gamma}\right)+\tilde{r}(x) \hat{z}^{\gamma}+r(x) \hat{y}^{\gamma}=\hat{\omega}^{\gamma} \chi_{\mathcal{O}}
\end{array} \quad \text { in } \quad L^{2}(0, T, \mathcal{O}) .\right.
$$

From (4.6) and (4.7), we get

$$
\hat{y}^{\gamma}(x, 0)=0, \hat{z}^{\gamma}(x, 0)=0 .
$$

Now, we have to prove that $\left(\zeta_{n}^{\gamma}, \xi_{n}^{\gamma}\right)$ converges to $\left(\hat{\zeta}^{\gamma}, \hat{\xi}^{\gamma}\right)$. Let $\zeta_{n}^{\gamma}=\zeta\left(\omega_{n}^{\gamma}\right)$ and $\xi_{n}^{\gamma}=\xi\left(\omega_{n}^{\gamma}\right)$. Reverse time variable by taking $\tilde{\zeta}_{n}^{\gamma}(x, t)=\zeta_{n}^{\gamma}(x, T-t), \quad \tilde{\xi}_{n}^{\gamma}(x, t)=\xi_{n}^{\gamma}(x, T-t), \quad \tilde{y}_{n}^{\gamma}(x, t)=y_{n}^{\gamma}(x, T-t)$ and $\tilde{z}_{n}^{\gamma}(x, t)=$ $z_{n}^{\gamma}(x, T-t)$. Then, we have

$$
\left\{\begin{array}{cc}
-\frac{\partial \tilde{\zeta}_{n}^{\gamma}}{\partial t}-\operatorname{div}\left(d(x) \nabla \tilde{\zeta}_{n}^{\gamma}\right)+r(x) \tilde{\zeta}_{n}^{\gamma}+r(x) \tilde{\xi}_{n}^{\gamma}=\tilde{y}_{n}^{\gamma} & \text { in } \quad Q, \\
-\frac{\partial \tilde{\xi}_{n}^{\gamma}}{\partial t}-\operatorname{div}\left(d(x) \nabla \tilde{\xi}_{n}^{\gamma}\right)+\tilde{r}(x) \tilde{\xi}_{n}^{\gamma}=\tilde{z}_{n}^{\gamma} & \text { in } \Omega, \\
\tilde{\zeta}_{n}^{\gamma}(x, 0)=0, \tilde{\xi}_{n}^{\gamma}(x, 0)=0 & \text { on } \Sigma . \\
\tilde{\zeta}_{n}^{\gamma}=0, \frac{\partial \tilde{\zeta}_{n}^{\gamma}}{\partial \nu}=0 &
\end{array}\right.
$$

Then, we deduce that

$$
\tilde{\zeta}_{n}^{\gamma} \rightarrow \hat{\zeta}^{\gamma} \text { weakly in } L^{2}(Q), \tilde{\xi}_{n}^{\gamma} \rightarrow \hat{\xi}^{\gamma} \text { weakly in } L^{2}\left(0, T ; H_{0}^{1}(\Omega)\right)
$$

Hence,

$$
\tilde{\zeta}_{n}^{\gamma}(x, 0) \rightarrow \hat{\zeta}^{\gamma}(x, 0) \text { weakly in } H^{-\frac{1}{2}}(\Omega), \tilde{\xi}_{n}^{\gamma}(x, 0) \rightarrow \hat{\xi}^{\gamma}(x, 0) \text { weakly in } H_{0}^{1}(\Omega)
$$


At last, we have

$$
\lim _{n \rightarrow \infty} \mathcal{J}^{\gamma}\left(\omega_{n}^{\gamma}\right)=\mathcal{J}^{\gamma}\left(\omega^{\gamma}\right)=\inf _{\omega \in \mathcal{U}_{a d}} \mathcal{J}^{\gamma}(\omega)
$$

The functional $\mathcal{J}^{\gamma}$ is quadratic coercive, thus $\hat{\omega}^{\gamma}$ is unique.

The characterization of the sequence of least regret controls is given in the following Proposition 4.1.

Proposition 4.1. The unique sequence of least regret controls $\hat{\omega}^{\gamma}$ is characterized by the following coupled system

$$
\begin{aligned}
& \left\{\begin{array}{cc}
\frac{\partial \hat{y}^{\gamma}}{\partial t}-\mathrm{d} i v\left(d(x) \nabla \hat{y}^{\gamma}\right)+r(x) \hat{y}^{\gamma}=0 & \\
\frac{\partial \hat{z}^{\gamma}}{\partial t}-\mathrm{d} i v\left(d(x) \nabla \hat{z}^{\gamma}\right)+\tilde{r}(x) \hat{z}^{\gamma}+r(x) \hat{y}^{\gamma}=\hat{\omega}^{\gamma} \chi_{\mathcal{O}} & \text { in } \quad Q \\
\hat{y}^{\gamma}(x, 0)=0, \hat{z}^{\gamma}(x, 0)=0, & \text { in } \quad \Omega \\
\hat{z}^{\gamma}=0, \frac{\partial \hat{z}^{\gamma}}{\partial \nu}=0 & \text { on } \quad \Sigma
\end{array}\right. \\
& \left\{\begin{array}{cc}
-\frac{\partial \hat{\zeta}^{\gamma}}{\partial t}-\mathrm{d} i v\left(d(x) \nabla \hat{\zeta}^{\gamma}\right)+r(x) \hat{\zeta}^{\gamma}+r(x) \hat{\xi}^{\gamma}=y\left(\omega-\hat{\omega}^{\gamma}\right) & \text { in } \quad Q \\
-\frac{\partial \hat{\xi}^{\gamma}}{\partial t}-\mathrm{d} i v\left(d(x) \nabla \hat{\xi}^{\gamma}\right)+\tilde{r}(x) \hat{\xi}^{\gamma}=z\left(\omega-\hat{\omega}^{\gamma}\right) & \text { in } \quad \Omega, \\
\hat{\zeta}^{\gamma}(x, T)=0, \hat{\xi}^{\gamma}(x, T)=0 & \text { on } \Sigma,
\end{array}\right. \\
& \left\{\begin{array}{cc}
\frac{\partial \hat{\rho}^{\gamma}}{\partial t}-\mathrm{d} i v\left(d(x) \nabla \hat{\rho}^{\gamma}\right)+r(x) \hat{\rho}^{\gamma}=0 & \text { in } \quad Q \\
\frac{\partial \hat{\sigma}^{\gamma}}{\partial t}-\mathrm{d} i v\left(d(x) \nabla \hat{\sigma}^{\gamma}\right)+\tilde{r}(x) \hat{\sigma}^{\gamma}+r(x) \hat{\rho}^{\gamma}=0 & \\
\hat{\rho}^{\gamma}(x, 0)=-\frac{1}{\gamma} \zeta\left(\omega-\hat{\omega}^{\gamma}\right)(x, 0), \hat{\sigma}^{\gamma}(x, 0)=-\frac{1}{\gamma} \xi\left(\omega-\hat{\omega}^{\gamma}\right)(x, 0) & \text { in } \quad \Omega, \\
\hat{z}^{\gamma}=0, \frac{\partial \hat{z}^{\gamma}}{\partial \nu}=0 & \text { on } \quad \Sigma,
\end{array}\right.
\end{aligned}
$$

and

$$
\left\{\begin{array}{cc}
-\frac{\partial \hat{p}^{\gamma}}{\partial t}-\mathrm{d} i v\left(d(x) \nabla \hat{p}^{\gamma}\right)+r(x) \hat{p}^{\gamma}+r(x) \hat{q}^{\gamma}=\hat{y}^{\gamma}-y_{d}+\hat{\rho}^{\gamma} & \text { in } \quad Q, \\
-\frac{\partial \hat{q}^{\gamma}}{\partial t}-\mathrm{d} i v\left(d(x) \nabla \hat{q}^{\gamma}\right)+\tilde{r}(x) \hat{q}^{\gamma}=\hat{z}^{\gamma}-z_{d}+\hat{\sigma}^{\gamma} & \text { in } \Omega, \\
\hat{p}^{\gamma}(x, T)=0, \hat{q}^{\gamma}(x, T)=0 & \text { on } \Sigma .
\end{array}\right.
$$

Furthermore, for all $\omega \in \mathcal{U}_{a d}$, we have

$$
\int_{0}^{T} \int_{\mathcal{O}}\left(\hat{q}^{\gamma}+N \hat{\omega}^{\gamma}\right)\left(\omega-\hat{\omega}^{\gamma}\right) \mathrm{d} x \mathrm{~d} t \geq 0 .
$$

Proof. The functional $\mathcal{J}^{\gamma}$ is quadratic coercive, thus it possesses a unique minimum $\hat{\omega}^{\gamma}$. This minimum is a solution to the Euler equation, thus for all $\omega \in \mathcal{U}_{a d}$, we have

$$
\lim _{h \rightarrow 0} \frac{J^{\gamma}\left(\hat{\omega}^{\gamma}+h\left(\omega-\hat{\omega}^{\gamma}\right)\right)-J^{\gamma}\left(\hat{\omega}^{\gamma}\right)}{h} \geq 0 .
$$


So, we have

$$
\begin{aligned}
\iint_{Q}\left[y\left(\omega-\hat{\omega}^{\gamma}\right)\left(\hat{y}^{\gamma}-y_{d}\right)+z(\omega\right. & \left.\left.-\hat{\omega}^{\gamma}\right)\left(\hat{z}^{\gamma}-z_{d}\right)\right] \mathrm{d} t \mathrm{~d} x+\int_{0}^{T} \int_{\mathcal{O}} N \hat{\omega}^{\gamma}\left(\omega-\hat{\omega}^{\gamma}\right) \mathrm{d} t \mathrm{~d} x \\
& +\frac{1}{\gamma} \int_{\Omega}\left[\hat{\zeta}^{\gamma}(x, 0) \zeta\left(\omega-\hat{\omega}^{\gamma}\right)(x, 0)+\hat{\xi}^{\gamma}(x, 0) \xi\left(\omega-\hat{\omega}^{\gamma}\right)(x, 0)\right] \mathrm{d} x \geq 0 .
\end{aligned}
$$

We introduce $\left(\hat{\rho}^{\gamma}, \hat{\sigma}^{\gamma}\right)=\left(\rho\left(\hat{\omega}^{\gamma}, 0\right)(x, t), \sigma\left(\hat{\omega}^{\gamma}, 0\right)(x, t)\right)$ solution to $(4.10)$. By integration by parts, we get

$$
\begin{aligned}
\iint_{Q} \hat{\zeta}^{\gamma}\left(\frac{\partial \hat{\rho}^{\gamma}}{\partial t}\right. & \left.-\operatorname{div}\left(d(x) \nabla \hat{\rho}^{\gamma}\right)+r(x) \hat{\rho}^{\gamma}\right) \mathrm{d} t \mathrm{~d} x \\
& =\iint_{Q} \hat{\rho}^{\gamma}\left(-\frac{\partial \hat{\zeta}^{\gamma}}{\partial t}-\operatorname{div}\left(d(x) \nabla \hat{\zeta}^{\gamma}\right)+r(x) \hat{\zeta}^{\gamma}\right) \mathrm{d} t \mathrm{~d} x+\frac{1}{\gamma} \int_{\Omega} \hat{\zeta}^{\gamma}(x, 0) \zeta\left(\omega-\hat{\omega}^{\gamma}\right)(x, 0) \mathrm{d} x=0
\end{aligned}
$$

and

$$
\begin{aligned}
\iint_{Q} \hat{\xi}^{\gamma}\left(\frac{\partial \hat{\sigma}^{\gamma}}{\partial t}-\operatorname{div}\left(d(x) \nabla \hat{\sigma}^{\gamma}\right)+\tilde{r}(x) \hat{\sigma}^{\gamma}\right. & \left.+r(x) \hat{\rho}^{\gamma}\right) \mathrm{d} t \mathrm{~d} x=\iint_{Q} \hat{\sigma}^{\gamma} z\left(\omega-\hat{\omega}^{\gamma}\right) \mathrm{d} t \mathrm{~d} x \\
& +\iint_{Q} \hat{\rho}^{\gamma} \tilde{r}(x) \hat{\xi}^{\gamma} \mathrm{d} t \mathrm{~d} x+\frac{1}{\gamma} \int_{\Omega} \hat{\xi}^{\gamma}(x, 0) \xi\left(\omega-\hat{\omega}^{\gamma}\right)(x, 0) \mathrm{d} x=0 .
\end{aligned}
$$

Adding (4.14) to (4.15) amounts to

$$
\begin{aligned}
\frac{1}{\gamma} \int_{\Omega}\left[\hat{\zeta}^{\gamma}(x, 0) \zeta\left(\omega-\hat{\omega}^{\gamma}\right)(x, 0)+\hat{\xi}^{\gamma}(x, 0) \xi\left(\omega-\hat{\omega}^{\gamma}\right)(x, 0)\right] \mathrm{d} x & \\
& =\iint_{Q}\left[\hat{\rho}^{\gamma} y\left(\omega-\hat{\omega}^{\gamma}\right)+\hat{\sigma}^{\gamma} z\left(\omega-\hat{\omega}^{\gamma}\right)\right] \mathrm{d} t \mathrm{~d} x
\end{aligned}
$$

Replacing (4.16) in (4.13), we find

$$
\begin{aligned}
\iint_{Q}\left[y\left(\omega-\hat{\omega}^{\gamma}\right)\left(\hat{y}^{\gamma}-y_{d}+\hat{\rho}^{\gamma}\right)+z\left(\omega-\hat{\omega}^{\gamma}\right)\left(\hat{z}^{\gamma}-z_{d}+\hat{\sigma}^{\gamma}\right)\right] \mathrm{d} t \mathrm{~d} x & \\
& +\int_{0}^{T} \int_{\mathcal{O}} N \hat{\omega}^{\gamma}\left(\omega-\hat{\omega}^{\gamma}\right) \mathrm{d} t \mathrm{~d} x \geq 0 .
\end{aligned}
$$

We introduce now the coupled adjoint state $\left(\hat{p}^{\gamma}, \hat{q}^{\gamma}\right)=\left(p\left(\hat{\omega}^{\gamma}, 0\right)(x, t), q\left(\hat{\omega}^{\gamma}, 0\right)(x, t)\right)$ solution to (4.11). Finally, we obtain (4.12) by replacing (4.11) in (4.17) and integration by parts.

We need some a priori estimations, which we make in the following Lemma.

Lemma 4.3. There exist some positive constants $\mathcal{C}$ independent of $\gamma$ satisfy the following estimations:

$$
\begin{aligned}
\left\|\hat{\omega}^{\gamma}\right\|_{L^{2}(0, T, \mathcal{O})} \leq \mathcal{C},\left\|\hat{y}^{\gamma}\right\|_{L^{2}(Q)} \leq \mathcal{C},\left\|\hat{z}^{\gamma}\right\|_{L^{2}(Q)} & \leq \mathcal{C} \\
& \frac{1}{\sqrt{\gamma}}\left\|\hat{\zeta}^{\gamma}(x, 0)\right\|_{H^{-\frac{1}{2}}(\Omega)} \leq \mathcal{C}, \frac{1}{\sqrt{\gamma}}\left\|\hat{\xi}^{\gamma}(x, 0)\right\|_{H_{0}^{1}(\Omega)} \leq \mathcal{C}
\end{aligned}
$$


and,

$$
\begin{aligned}
&\left\|\hat{y}^{\gamma}\right\|_{L^{2}(Q)} \leq \mathcal{C},\left\|\frac{\partial \hat{y}^{\gamma}}{\partial t}\right\|_{L^{2}(Q)} \leq \mathcal{C},\left\|\hat{z}^{\gamma}\right\|_{L^{2}\left(0, T ; H_{0}^{1}(\Omega)\right)} \leq \mathcal{C},\left\|\frac{\partial \hat{z}^{\gamma}}{\partial t}\right\|_{L^{2}(Q)} \leq \mathcal{C}, \\
&\left\|\hat{\zeta}^{\gamma}\right\|_{L^{2}(Q)} \leq \mathcal{C},\left\|\frac{\partial \hat{\zeta}^{\gamma}}{\partial t}\right\|_{L^{2}(Q)} \leq \mathcal{C},\left\|\hat{\xi}^{\gamma}\right\|_{L^{2}\left(0, T ; H_{0}^{1}(\Omega)\right)} \leq \mathcal{C},\left\|\frac{\partial \hat{\xi}^{\gamma}}{\partial t}\right\|_{L^{2}(Q)} \leq \mathcal{C}, \\
&\left\|\hat{\rho}^{\gamma}\right\|_{L^{2}(Q)} \leq \mathcal{C},\left\|\frac{\partial \hat{\rho}^{\gamma}}{\partial t}\right\|_{L^{2}(Q)} \leq \mathcal{C},\left\|\hat{\sigma}^{\gamma}\right\|_{L^{2}\left(0, T ; H_{0}^{1}(\Omega)\right)} \leq \mathcal{C},\left\|\frac{\partial \hat{\sigma}^{\gamma}}{\partial t}\right\|_{L^{2}(Q)} \leq \mathcal{C}, \\
&\left\|\hat{p}^{\gamma}\right\|_{L^{2}(Q)} \leq \mathcal{C},\left\|\frac{\partial \hat{p}^{\gamma}}{\partial t}\right\|_{L^{2}(Q)} \leq \mathcal{C},\left\|\hat{q}^{\gamma}\right\|_{L^{2}\left(0, T ; H_{0}^{1}(\Omega)\right)} \leq \mathcal{C},\left\|\frac{\partial \hat{q}^{\gamma}}{\partial t}\right\|_{L^{2}(Q)} \leq \mathcal{C} .
\end{aligned}
$$

Proof. Since $\hat{\omega}^{\gamma}$ is the sequence of least regret controls, we have

$$
\mathcal{J}^{\gamma}\left(\hat{\omega}^{\gamma}\right) \leq \mathcal{J}^{\gamma}(\omega) \quad \forall \omega \in \mathcal{U}_{a d}
$$

In particular case when $\omega=0$, we get

$$
J\left(\hat{\omega}^{\gamma}, 0\right)-J(0,0)+\frac{1}{\gamma}\left\|\hat{\zeta}^{\gamma}(x, 0)\right\|_{H^{-\frac{1}{2}}(\Omega)}^{2}+\frac{1}{\gamma}\left\|\hat{\xi}^{\gamma}(x, 0)\right\|_{H_{0}^{1}(\Omega)}^{2} \leq 0 .
$$

Thus, we have

$$
\begin{array}{r}
\left\|\hat{y}^{\gamma}-y_{d}\right\|_{L^{2}(Q)}^{2}+\left\|\hat{z}^{\gamma}-z_{d}\right\|_{L^{2}(Q)}^{2}+N\left\|\mid \hat{\omega}^{\gamma}\right\|_{L^{2}(Q)}^{2}+\frac{1}{\gamma}\left\|\hat{\zeta}^{\gamma}(x, 0)\right\|_{H^{-\frac{1}{2}}(\Omega)}^{2}+\frac{1}{\gamma}\left\|\hat{\xi}^{\gamma}(x, 0)\right\|_{H_{0}^{1}(\Omega)}^{2} \\
\leq\left\|y_{d}\right\|_{L^{2}(\Omega)}^{2}+\left\|z_{d}\right\|_{L^{2}(\Omega)}^{2}=\text { Constant }
\end{array}
$$

So (4.18) holds. Multiplying the first equality of (4.8) by $\hat{y}^{\gamma}$ and the second equality by $\hat{z}^{\gamma}$ and we integrate over $\Omega$, we find

$$
\begin{aligned}
\int_{\Omega} \hat{y}^{\gamma}(t)\left(\frac{\partial \hat{y}^{\gamma}(t)}{\partial t}-\operatorname{div}(d(x)\right. & \left.\left.\nabla \hat{y}^{\gamma}(t)\right)+r(x) \hat{y}^{\gamma}(t)\right) \mathrm{d} x \\
& =\frac{1}{2} \frac{\mathrm{d}}{\mathrm{d} t} \int_{\Omega}\left|\hat{y}^{\gamma}(t)\right|^{2} \mathrm{~d} x+\int_{\Omega} r(x)\left|\hat{y}^{\gamma}(t)\right|^{2} \mathrm{~d} x-\int_{\Omega} \mathrm{d} i v\left(d(x) \hat{y}^{\gamma}(t)\right) \hat{y}^{\gamma}(t) \mathrm{d} x=0
\end{aligned}
$$

and

$$
\begin{aligned}
& \int_{\Omega} \hat{z}^{\gamma}(t)\left(\frac{\partial \hat{z}^{\gamma}(t)}{\partial t}-\operatorname{div}\left(d(x) \nabla \hat{z}^{\gamma}(t)\right)+\tilde{r}(x) \hat{z}^{\gamma}(t)+r(x) \hat{y}^{\gamma}(t)\right) \mathrm{d} x \\
&=\frac{1}{2} \frac{\mathrm{d}}{\mathrm{d} t} \int_{\Omega}\left|\hat{z}^{\gamma}(t)\right|^{2} \mathrm{~d} x+\int_{\Omega} \tilde{r}(x)\left|\hat{z}^{\gamma}(t)\right|^{2} \mathrm{~d} x-\int_{\Omega} \mathrm{d} i v\left(d(x) \cdot \hat{z}^{\gamma}(t)\right) \hat{z}^{\gamma}(t)+\int_{\Omega} r(x) \hat{y}^{\gamma}(t) \hat{z}^{\gamma}(t) \mathrm{d} x \\
&=\int_{\mathcal{O}} \hat{\omega}^{\gamma}(t) \hat{z}^{\gamma}(t) \mathrm{d} x .
\end{aligned}
$$

By integrating over $[0, T]$ and by applying the Gronwall lemma we obtain

$$
\left\|\hat{y}^{\gamma}\right\|_{L^{2}(Q)} \leq \mathcal{C}_{\gamma},\left\|\frac{\partial \hat{y}^{\gamma}}{\partial t}\right\|_{L^{2}(Q)} \leq \mathcal{C}_{\gamma},\left\|\hat{z}^{\gamma}\right\|_{L^{2}\left(0, T ; H_{0}^{1}(\Omega)\right)} \leq \mathcal{C}_{\gamma},\left\|\frac{\partial \hat{z}^{\gamma}}{\partial t}\right\|_{L^{2}(Q)} \leq \mathcal{C}_{\gamma}
$$

where $\mathcal{C}_{\gamma}$ is a positive constant. From the last estimations we get (4.19). We follow a similar method to demonstrate (4.19) for finding (4.20). 
When we pass to the limit when $\gamma \rightarrow 0$, the sequence of least regret controls converges $\hat{\omega}^{\gamma}$ to the no regret control $\hat{\omega}$.

Theorem 4.1. The no regret control $\hat{\omega}=\lim _{\gamma \rightarrow 0} \hat{\omega}^{\gamma}$ is characterized by the unique set $\{(\hat{y}, \hat{z}),(\hat{\zeta}, \hat{\xi}),(\hat{\rho}, \hat{\sigma}),(\hat{p}, \hat{q})\}$ solution to the following coupled optimality system (SO)

$$
\left\{\begin{array}{cc}
\frac{\partial \hat{\rho}}{\partial t}-\mathrm{d} i v(d(x) \nabla \hat{\rho})+r(x) \hat{\rho}=0 & \text { in } \quad Q \\
\frac{\partial \hat{\sigma}}{\partial t}-\mathrm{d} i v(d(x) \nabla \hat{\sigma})+\tilde{r}(x) \hat{\sigma}+r(x) \hat{\rho}=0 & \\
\hat{\rho}(x, 0)=\rho_{0}, \hat{\sigma}(x, 0)=\sigma_{0} & \text { in } \quad \Omega, \\
\hat{\sigma}=0, \frac{\partial \hat{\sigma}}{\partial \nu}=0 & \text { on } \quad \Sigma
\end{array}\right.
$$

and

$$
\left\{\begin{array}{cc}
-\frac{\partial \hat{p}}{\partial t}-\mathrm{d} i v(d(x) \nabla \hat{p})+r(x) \hat{p}+r(x) \hat{q}=\hat{y}-y_{d}+\hat{\rho} & \text { in } \quad Q \\
-\frac{\partial \hat{q}}{\partial t}-\mathrm{d} i v(d(x) \nabla \hat{q})+\tilde{r}(x) \hat{q}=\hat{z}-z_{d}+\hat{\sigma} & \text { in } \quad \Omega \\
\hat{p}(x, T)=0, \hat{q}(x, T)=0 & \text { on } \quad \Sigma
\end{array}\right.
$$

with the variational inequality

$$
\int_{0}^{T} \int_{\mathcal{O}}(\hat{q}+N \hat{\omega})(\omega-\hat{\omega}) \mathrm{d} x \mathrm{~d} t \geq 0
$$

with the following limits:

$$
\rho_{0}=-\lim _{\gamma \rightarrow 0} \frac{1}{\gamma} \zeta\left(\omega-\hat{\omega}^{\gamma}\right)(x, 0), \sigma_{0}=-\lim _{\gamma \rightarrow 0} \frac{1}{\gamma} \xi\left(\omega-\hat{\omega}^{\gamma}\right)(x, 0) .
$$

Proof. From inequality (4.23), we may extract some some subsequence of $\left(\hat{\omega}^{\gamma}, \hat{y}^{\gamma}, \hat{z}^{\gamma}\right)_{\gamma}$ that we denote with the same indices such that, when $\gamma$ goes to 0 , we have

$$
\hat{\omega}^{\gamma} \rightarrow \hat{\omega} \quad \text { weakly in } L^{2}(0, T ; \mathcal{O}), \quad\left(\hat{y}^{\gamma}, \hat{z}^{\gamma}\right) \rightarrow(\hat{y}, \hat{z}) \quad \text { weakly in } \quad L^{2}(Q) \times L^{2}\left(0, T ; H_{0}^{1}(\Omega)\right)
$$


On the other hand, from the optimality coupled systems in Proposition 4.1, the sequences $\left(\frac{\partial \hat{y}^{\gamma}}{\partial t}-\operatorname{div}\left(d(x) \nabla \hat{y}^{\gamma}\right)+\right.$ $\left.r(x) \hat{y}^{\gamma}\right)_{\gamma}$ and $\left(\frac{\partial \hat{z}^{\gamma}}{\partial t}-\operatorname{div}\left(d(x) \nabla \hat{z}^{\gamma}\right)+\tilde{r}(x) \hat{z}^{\gamma}+r(x) \hat{y}^{\gamma}\right)_{\gamma}$ are bounded in $L^{2}(Q)$. So, we have

$$
\begin{gathered}
\frac{\partial \hat{y}^{\gamma}}{\partial t}-\operatorname{div}\left(d(x) \nabla \hat{y}^{\gamma}\right)+r(x) \hat{y}^{\gamma} \rightarrow \frac{\partial \hat{y}}{\partial t}-\operatorname{div}(d(x) \nabla \hat{y})+r(x) \hat{y} \text { weakly in } L^{2}(Q), \\
\frac{\partial \hat{z}^{\gamma}}{\partial t}-\operatorname{div}\left(d(x) \nabla \hat{z}^{\gamma}\right)+\tilde{r}(x) \hat{z}^{\gamma}+r(x) \hat{y}^{\gamma} \rightarrow \frac{\partial \hat{z}}{\partial t}-\operatorname{div}(d(x) \nabla \hat{z})+\tilde{r}(x) \hat{z}+r(x) \hat{y} \text { in } L^{2}(Q) .
\end{gathered}
$$

Taking to the limit $\gamma \rightarrow 0$, we get (4.24). Also, from to a priori estimates of Lemma 4.3, and by using the same method we found (4.25)-(4.27). From (4.18), we have

$$
-\frac{1}{\gamma}\left(\zeta\left(\omega-\hat{\omega}^{\gamma}\right)(x, 0), \xi\left(\omega-\hat{\omega}^{\gamma}\right)(x, 0)\right) \rightarrow\left(\rho_{0}, \sigma_{0}\right) \text { weakly in } H^{-\frac{1}{2}}(\Omega) \times H_{0}^{1}(\Omega) .
$$

In close, the inequality (4.28) can be deduced using the weak convergence of $\hat{p}^{\gamma}, \hat{q}^{\gamma}$ and $\hat{\omega}^{\gamma}$.

\section{Conclusion}

This work examined an evolution coupled system, with missing initial conditions that presented the relation between biochemical oxygen demand (BOD) and dissolved oxygen (DO). Since the decrease of BOD is a good way for judging the effectiveness of water purification, our main objective was to control the level of dissolved oxygen, for giving more information about it. We gave the characterization of the optimal control through applied the idea of no regret, where we solved an optimal control problem with uncertainly. The obtained method optimization problem is to be transformed into classical optimal control via the notion of low regret control. Finally, the coupled optimality system for the least regret control converges weakly to the coupled optimality systems for no regret control or the optimal control.

Conflicts of Interest: The author(s) declare that there are no conflicts of interest regarding the publication of this paper.

\section{REFERENCES}

[1] J. J. Mocuba, Dissolved oxygen and biochemical oxygen demand in the waters close to the Quelimane sewage discharge. MS Thesis. The University of Bergen (2010).

[2] J. Liu, G. Olsson, B. Mattiasson, Short-term BOD (BODst) as a parameter for on-line monitoring of biological treatment process, Biosensors Bioelectron. 20 (2004), 562-570.

[3] J. Liu, B. Mattiasson, Microbial BOD sensors for wastewater analysis, Water Res. 36 (2002), 3786-3802.

[4] J. L. Lions Contrôle de Pareto de systèmes distribués. Le cas stationnaire C. R. Acad. Sci. Paris Sér. I, 302 (6) (1986), $223-227$.

[5] J. L. Lions, Control of Distributed Systems with incomplete Data. AMS Colloquium, Berkeley, 1983.

[6] J. L. Lions Contrôle à moindres regrets des systèmes distribuès. C. R. Acad. Sci. Paris Sér. I, 315(12) (1992), $1253-1257$.

[7] J. L. Lions, No-regret and low-regret control, Environment, Economics and Their Mathematical Models, Masson, Paris, (1994). 
[8] R. Dorville, O. Nakoulima, A. Omrane, On the control of ill-posed distributed parametersystems, ESAIM: Proc. 17 (2007), $50-66$.

[9] R. Dorville, O. Nakoulima, A. Omrane, Contrôle optimal pour les problèmes de contrôlabilité des systèmes distribués à données manquantes, C. R. Math. Acad. Sci. Paris, Ser. I, 338(12) (2004), 921-924.

[10] O. Nakoulima, A. Omrane, J. Velin, No-regret control for nonlinear distributed systems with incomplete data, J. Math. Pures Appl. 81 (2002), 1161-1189.

[11] A. Hafdallah, A. Ayadi, Optimal control of a thermoelastic body with missing initial conditions, Int. J. Control. 93 (2020), $1570-1576$.

[12] D. M. Reynolds, S. R. Ahmad, Rapid and direct determination of wastewater BOD values using a fluorescence technique, Water Res. 31 (1997), 2012-2018.

[13] J. Salguero, J. Valverde Flores, Reduction of the Biochemical Oxygen Demand of the water samples from the lower basin of the Chillon River by means of Air-Ozone MicroNanobubbles, Ventanilla - Callao, J. Nanotechnol. (Lima). 1 (2017), 25-35.

[14] M. Zajda, U. Aleksander-Kwaterczak, Wastewater Treatment Methods for Effluents from the Confectionery Industry - an Overview, J. Ecol. Eng. 20 (2019), 293-304.

[15] A. Hafdallah, A. Ayadi, C. Laouar, No-Regret Optimal Control Characterization for an Ill-Posed Wave Equation, Int. J. Math. Trends Techno. 41 (2017), 1-6. 